\title{
Experimental Realization of a Quantum Phase Transition of Polaritonic Excitations
}

\author{
Kenji Toyoda, ${ }^{*}$ Yuta Matsuno, Atsushi Noguchi, Shinsuke Haze, ${ }^{\dagger}$ and Shinji Urabe \\ Graduate School of Engineering Science, Osaka University, 1-3 Machikaneyama, Toyonaka, Osaka, Japan
}

(Received 9 July 2013; published 14 October 2013)

\begin{abstract}
We report an experimental realization of the Jaynes-Cummings-Hubbard model using the internal and radial phonon states of two trapped ions. An adiabatic transfer corresponding to a quantum phase transition from a localized insulator ground state to a delocalized superfluid (SF) ground state is demonstrated. The SF phase of polaritonic excitations characteristic of the interconnected JaynesCummings (JC) system is experimentally explored, where a polaritonic excitation refers to a combination of an atomic excitation and a phonon interchanged via a JC coupling.
\end{abstract}

The Jaynes-Cummings (JC) model [1,2] describing the interaction between a quantized optical mode and a twolevel atom is one of the simplest and most important models of light-matter interactions. An interconnected array of multiple JC systems has recently been attracting interest, and the model describing it is referred to as the Jaynes-Cummings-Hubbard (JCH) model [3-9]; an experimental realization of this model has remained to be done. The JCH model was originally proposed for an array of coupled optical cavities, each containing a two-level atom, and is expected to exhibit properties peculiar to strongly correlated systems [10-12].

In the JCH model for an array of coupled optical cavities, photons naturally hop between neighboring cavities, whereas the photon-photon interaction arises from a photon blockade [13], which impedes other photons from entering an occupied cavity.

The JCH model has certain similarities to the BoseHubbard (BH) model [11,12]. It approaches the pure bosonic case in the large detuning and the large photon number limits [3]. In contrast, in the limit of small detuning and small phonon numbers, the coefficient for the on-site repulsion becomes dependent on the photon number. In addition, the conserved particles (polaritons or dressed atoms) transform into various kinds of excitations (atomic excitations, photons, or polaritons) depending on the Rabi frequency and detuning. As a result, a JCH system has a richer phase structure compared with a BH system. Both photons and polaritons can show superfluidity, while insulator phases can be formed with both atoms and polaritons [6,7].

Recent advances in the ability to manipulate quantum systems have made it possible to simulate a quantum system using another controllable system (analog quantum computation) [14]. Trapped ions offer high controllability and individual access, and hence are suited for such applications $[15,16]$. Simulations of systems including spin systems and relativistic electrons have been reported [17-21]. Simulations of Hubbard models have also been proposed for trapped ions [9,22]; however, an experimental demonstration has remained to be done.
The phonons in the radial (or transverse) direction of a linear ionic chain, which have been used to mediate spinspin interactions [19,21], can also be used to simulate systems of Bosonic particles under certain conditions [22]. In contrast to the axial motion of ions in a linear chain, which is described by collective modes that span over the whole ionic chain, radial phonons under a sufficiently tight radial confinement are essentially 'local phonons' (phonons of local harmonic oscillations) undergoing hopping from site to site with a rate slower than the local harmonic-oscillation frequencies. We recently observed this hopping of radial phonons using two trapped ions [23]. Ivanov et al. [9] proposed to use a JC coupling arising from optical excitation of the radial red-sideband transition of a linear ionic chain to induce an effective phononphonon coupling, thereby realizing the JCH model. In this Letter, we report an experimental realization of the JCH model and observation of a quantum phase transition based on Ivanov et al. [9] In this case the conserved particles are not merely phonons but composite particles each of which is a linear combination of a phonon and an atomic excitation.

The conceptual schematic of the JCH system using trapped ions is shown in Fig. 1(a). It is assumed that two ions with internal states $\left\{\left|g_{j}\right\rangle,\left|e_{j}\right\rangle\right\}$ and a resonance frequency $\omega_{0}$ are held in a linear Paul trap. Each of the ions undergoes harmonic motion in a radial direction (referred to as the $x$ direction). Both ions are equally illuminated with a laser of frequency $\omega_{L}$ and detuning $\delta=\omega_{L}-\omega_{0}$, which is nearly resonant with the radial red-sideband transitions. Then the system is approximately governed by the following Hamiltonian (a JCH Hamiltonian) [9]:

$$
\begin{aligned}
H= & \hbar \Delta \sum_{j=1,2}\left|e_{j}\right\rangle\left\langle e_{j}\right|+\hbar g \sum_{j=1,2}\left(\hat{a}_{j}^{\dagger} \hat{\sigma}_{j}^{-}+\hat{a}_{j} \hat{\sigma}_{j}^{+}\right) \\
& +\frac{\kappa}{2}\left(\hat{a}_{1}^{\dagger} \hat{a}_{2}+\hat{a}_{2}^{\dagger} \hat{a}_{1}\right) .
\end{aligned}
$$

Here $\Delta \equiv-\delta-\omega_{x}^{\prime}=-\left[\omega_{L}-\left(\omega_{0}-\omega_{x}^{\prime}\right)\right]$ is the detuning from the radial red-sideband transition, where $\omega_{x}^{\prime} \equiv$ $\omega_{x}+\Delta \omega_{x}$ is the oscillation frequency for the radial $x$ 
(a)

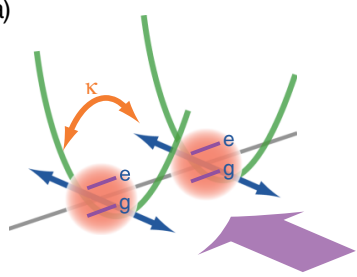

(b)

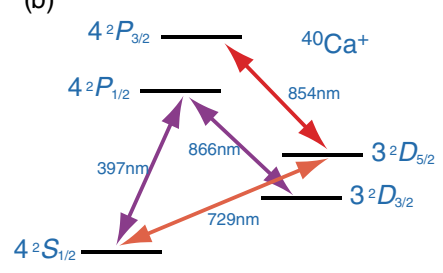

FIG. 1 (color online). (a) Conceptual schematic of the JCH system with two ions. Two ions are illuminated with an excitation laser nearly resonant to the red-sideband transition with which the JC coupling arises and dressed atoms or polaritons are formed. Intersite hopping $(\kappa)$ is naturally incorporated from Coulomb couplings, and along with effective on-site repulsion between polaritons due to the JC coupling a Hubbard-type model is formed. (b) Energy levels of ${ }^{40} \mathrm{Ca}^{+}$relevant to motional cooling and induction of the JC coupling.

direction, in which $\omega_{x}$ is the oscillation frequency of a single ion in the radial $x$ direction and $\Delta \omega_{x}=-\omega_{z}^{2} / 4 \omega_{x}=$ $-e^{2} / 8 \pi \epsilon_{0} d_{0}^{3} m \omega_{x}$ is the correction due to the Coulomb interaction ( $d_{0}$ is the interion distance and $m$ is the mass of one ion). $g \equiv \eta \Omega_{0} / 2$ is the coupling coefficient for the red-sideband transition (JC coupling) where $\eta$ is the Lamb-Dicke factor and $\Omega_{0}$ is the on-resonance Rabi frequency. $\hat{a}_{j}^{\dagger}$ and $\hat{a}_{j}$ are the creation and annihilation operators of phonons in the radial $x$ direction of the $j$ th ion, whose Hilbert space is spanned by Fock-state basis $|n\rangle_{j}(n=$ $0,1,2, \ldots) . \hat{\sigma}_{j}^{+} \equiv\left|e_{j}\right\rangle\left\langle g_{j}\right|$ and $\hat{\sigma}_{j}^{-} \equiv\left|g_{j}\right\rangle\left\langle e_{j}\right|$ are the raising and lowering operators for the internal states. $\kappa=$ $\omega_{z}^{2} / 2 \omega_{x}=e^{2} / 4 \pi \epsilon_{0} d_{0}^{3} m \omega_{x}$ is the hopping rate for the radial $x$ direction.

A JCH system is expected to show quantum phase transitions between superfluid and insulator phases of polaritons [5]. Here a 'superfluid' is a system that has delocalized excitations and in which there is a correlation between mechanical variables at different sites. On the other hand, an 'insulator' is a system that has localized excitations.

As an order parameter characterizing the quantum phase transition, the variance of the total excitation number per site $\Delta \hat{N}_{j}^{2} \equiv\left\langle\hat{N}_{j}^{2}\right\rangle-\left\langle\hat{N}_{j}\right\rangle^{2}$, where $\hat{N}_{j}=\hat{a}_{j}^{\dagger} \hat{a}_{j}+\left|e_{j}\right\rangle\left\langle e_{j}\right|$, can be used [5]. The expectation value of the annihilation operator that is usually used in the mean-field limit cannot be used as the order parameter, since it is always zero for a closed system with no particle exchange with the outside [5,7]. In addition, the atomic excitation number variance $\Delta \hat{N}_{a, j}^{2} \equiv\left\langle\hat{N}_{a, j}^{2}\right\rangle-\left\langle\hat{N}_{a, j}\right\rangle^{2}$, where $\hat{N}_{a, j}=\left|e_{j}\right\rangle\left\langle e_{j}\right|$, is also used for judging the existence of polaritons.

The experimental setup used is similar to that described in Ref. [23] and a brief description is given here. Two ${ }^{40} \mathrm{Ca}^{+}$ions are trapped in vacuum $\left(5 \times 10^{-9} \mathrm{~Pa}\right)$ using a linear Paul trap. A rf voltage of $25 \mathrm{MHz}$ is applied to generate the radial confinements and dc electrodes provide an axial confinement. The secular frequencies for the three trap axes are $\left(\omega_{x}, \omega_{y}, \omega_{z}\right) / 2 \pi=(2.1,1.7,0.17) \mathrm{MHz}$. The interion distance in the axial direction $d_{0}$ is $18-20 \mu \mathrm{m}$ and correspondingly, the hopping rate $\kappa / 2 \pi$ is $5-7 \mathrm{kHz}$.

The energy levels relevant to motional cooling and induction of the JC coupling are shown in Fig. 1(b). The motion in the radial directions is cooled by Doppler cooling using the $S_{1 / 2}-P_{1 / 2}(397 \mathrm{~nm})$ and $D_{3 / 2}-P_{1 / 2}(866 \mathrm{~nm})$ transitions and sideband cooling using the $S_{1 / 2}\left(m_{J}=-1 / 2\right)$ $D_{5 / 2}\left(m_{J}=-5 / 2\right)(729 \mathrm{~nm})$ and $D_{5 / 2}-P_{3 / 2}(854 \mathrm{~nm})$ transitions. There are two collective modes in the $x$ direction of two ions, namely the center-of-mass (c.m.; in-phase) mode and the rocking (out-of-phase) mode, just as in the case of the axial motion [24]. The average quantum numbers after the sideband cooling are $\left(\bar{n}_{x, \mathrm{c} . \mathrm{m} .}, \bar{n}_{x, \text { rock }}, \bar{n}_{y, \mathrm{c} . \mathrm{m} .}, \bar{n}_{y, \text { rock }}\right)=$ $(0.04 \pm 0.04,0.03 \pm 0.03,0.57 \pm 0.11,0.08 \pm 0.04)$. The axial motion is cooled only by Doppler cooling. The ions are intermittently optically pumped to $S_{1 / 2}\left(m_{J}=-1 / 2\right)$ by using a 397-nm beam with the $\sigma^{-}$polarization during and after the sideband cooling. The excitation beam at $729 \mathrm{~nm}$ for the $S_{1 / 2}-D_{5 / 2}$ transition, which is used to induce the JC coupling and other operations, is oriented at $45^{\circ}, 45^{\circ}$, and $90^{\circ}$ relative to the $x, y$, and $z$ directions, respectively. This direction is chosen to couple the beam only to the radial directions and to ignore the axial directions, whose secular frequency is relatively small and hence less advantageous in sideband cooling because of the large average quantum number after Doppler cooling. Equal illumination of the two ions with this beam is carefully optimized by adjusting the beam position so that the intensity difference between the two ions becomes less than $5 \%$.

The internal state of the ions is determined by illuminating them with lasers at $397 \mathrm{~nm}\left(S_{1 / 2}\right.$ to $P_{1 / 2}$ transition $)$ and $866 \mathrm{~nm}\left(D_{3 / 2}\right.$ to $P_{1 / 2}$ transition) and by detecting fluorescence photons with a photomultiplier or an intensified charge-coupled-device (ICCD) camera, with detection times of 8 and $80 \mathrm{~ms}$, respectively. Individual detection of fluorescence from each ion is possible with the ICCD camera. Due to unequal illumination intensity of the two ions with the 397-nm laser, individual detection is possible also when using the photomultiplier.

First, the dynamics of the JCH system with two ions is observed. The total dynamics of the two-ion JCH system arises from the JC coupling in individual atoms excited by the excitation laser and intersite hopping [23]. When the hopping rate $\kappa$ is much smaller than the JC coupling coefficient $g$, a simple sinusoidal oscillation similar to Rabi dynamics caused by the sideband excitation of the local radial oscillation modes is expected, while for nonnegligible values of $\kappa$, an interference between Rabi and hopping dynamics is expected. Figure 2 shows the result of the observation of the JCH dynamics for two ions (the circles), where the population of the internal state of each ion is plotted. The system is initially prepared in the $\left|g_{1}\right\rangle\left|g_{2}\right\rangle|0\rangle_{1}|0\rangle_{2}$ state with sideband cooling and optical pumping. The excitation laser is tuned to the resonance of the blue-sideband transition of the radial $x$ mode. This 
(a)

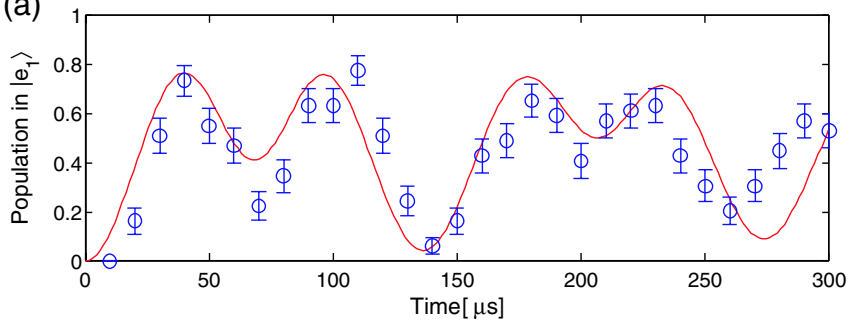

(b)

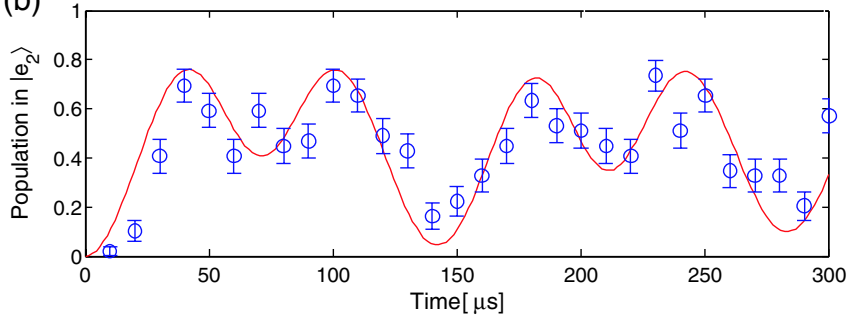

FIG. 2 (color online). Measured and simulated quantum dynamics of the JCH system with two ions. Populations of the excited state of one ion and the other are plotted in (a) and (b), respectively, with circles. Each point obtained is the average of 50 experiments. Curves are numerically simulated results, which are multiplied by 0.8 to consider population quenching from $D_{5 / 2}$ to $S_{1 / 2}$ in the relatively long detection time of $80 \mathrm{~ms}$ due to a stray intensity from the $854-\mathrm{nm}$ beam.

gives rise to an anti-Jaynes-Cummings coupling [2], which is formally equivalent to a JC coupling when the internal states $\left\{\left|g_{j}\right\rangle,\left|e_{j}\right\rangle\right\}$ are interchanged. The red curves show numerically simulated dynamics for the hopping rate $\kappa / 2 \pi$ of $5.4 \mathrm{kHz}$, the JC coupling coefficient $2 g / 2 \pi$ of $12.0 \mathrm{kHz}$, and the coherence relaxation due to laser frequency fluctuations of $200 \mathrm{~Hz}$. Although the dynamics is periodical, it is greatly modified from a simple sinusoidal oscillation due to the effect of the intersite hopping term. The two ions show almost the same dynamics as expected from equal illumination.

As a demonstration of a quantum phase transition, an adiabatic transfer from an insulator ground state to a superfluid (SF) ground state is observed in the average excited-state population of two ions [see Fig. 3(a)].

The transfer process starts from a point where $-\Delta / g$ is large, where the ground state is approximately $\left|\psi_{\text {atI }}\right\rangle \equiv$ $\left|e_{1}\right\rangle\left|e_{2}\right\rangle|0\rangle_{1}|0\rangle_{2}$ [the atomic insulator state (atI)]. Then $\Delta / g$ increases, exceeds zero, and becomes a large positive value, where the ground state is approximately $\left|\psi_{\mathrm{phSF}}\right\rangle \equiv$ $\left|g_{1}\right\rangle\left|g_{2}\right\rangle \otimes\left[(1 / \sqrt{2})|1\rangle_{1}|1\rangle_{2}-(1 / 2)|2\rangle_{1}|0\rangle_{2}-(1 / 2)|0\rangle_{1}|2\rangle_{2}\right]=$ $\left|g_{1}\right\rangle\left|g_{2}\right\rangle(1 / \sqrt{2}) \hat{a}_{r}^{\dagger 2}|0\rangle_{1}|0\rangle_{2}$ [the phonon SF state (phSF)]. Here, $\hat{a}_{r}^{\dagger}=(1 / \sqrt{2})\left(\hat{a}_{1}^{\dagger}-\hat{a}_{2}^{\dagger}\right)$ is the rocking-mode creation operator. This phase is the phonon SF phase. In the intermediate region around $\Delta \sim 0$, the system is in the polaritonic SF state. This polaritonic SF state is approximated as $(1 / \sqrt{3})\left|\psi_{\mathrm{phSF}}\right\rangle+(1 / \sqrt{6})\left|\psi_{\mathrm{atI}}\right\rangle+(1 / 2 \sqrt{2})\left(\left|e_{1}\right\rangle\left|g_{2}\right\rangle|1\rangle_{1}|0\rangle_{2}+\right.$ $\left.\left|g_{1}\right\rangle\left|e_{2}\right\rangle|0\rangle_{1}|1\rangle_{2}-\left|e_{1}\right\rangle\left|g_{2}\right\rangle|0\rangle_{1}|1\rangle_{2}-\left|g_{1}\right\rangle\left|e_{2}\right\rangle|1\rangle_{1}|0\rangle_{2}\right)[7]$.

In the experiment, $\left|\psi_{\text {atI }}\right\rangle$ is prepared with cooling, optical pumping, and applying a carrier $\pi$ pulse. Then the (a)

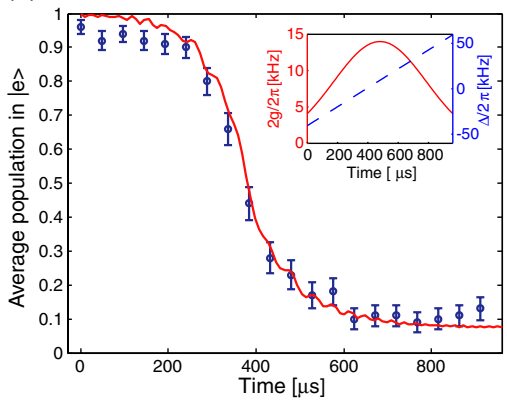

(b)

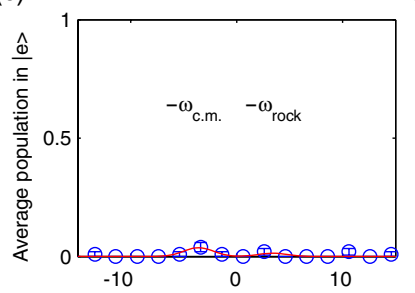

(e)

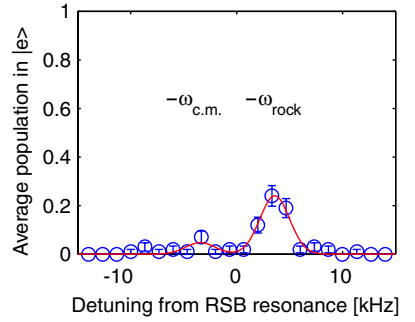

(d)

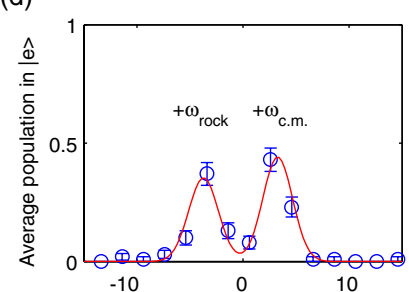

(f)

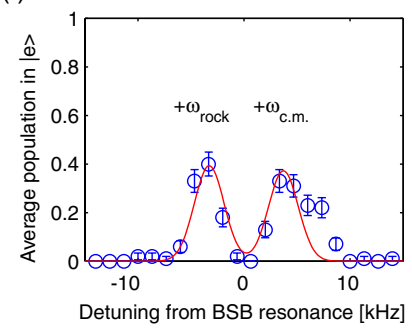

FIG. 3 (color online). (a) Variation of the average internal-state populations during the adiabatic transfer. Each point obtained is the average of 50 experiments. The inset shows the time dependence of the JC coupling coefficient $2 g / 2 \pi$ (the solid curve with the vertical axis on the left) and the detuning $\Delta / 2 \pi$ (the dashed curve with the vertical axis on the right). (b) Time dependence of the eigenenergies obtained by diagonalyzing the instantaneous Hamiltonians based on the pulse parameters used in the experiment. The lowest three curves corresponds, from the lowest to the third lowest, to $\left|\psi_{\text {atI }}\right\rangle \rightarrow\left|\psi_{\mathrm{phSF}}\right\rangle,(1 / \sqrt{2})\left(\left|g_{1}\right\rangle\left|e_{2}\right\rangle+\left|e_{1}\right\rangle\left|g_{2}\right\rangle\right) \otimes$ $\hat{a}_{r}^{\dagger}|0\rangle_{1}|0\rangle_{2} \rightarrow\left|g_{1}\right\rangle\left|g_{2}\right\rangle \otimes \hat{a}_{c}^{\dagger} \hat{a}_{r}^{\dagger}|0\rangle_{1}|0\rangle_{2}, \quad$ and $(1 / \sqrt{2})\left(\left|g_{1}\right\rangle\left|e_{2}\right\rangle-\right.$ $\left.\left|e_{1}\right\rangle\left|g_{2}\right\rangle\right) \otimes \hat{a}_{r}^{\dagger}|0\rangle_{1}|0\rangle_{2} \rightarrow\left|g_{1}\right\rangle\left|g_{2}\right\rangle \otimes(1 / \sqrt{2}) \hat{a}_{c}^{\dagger 2}|0\rangle_{1}|0\rangle_{2}$, respectively. (c),(d) Measurement of the average phonon numbers of collective modes before the adiabatic transfer. Results of spectroscopy over radial red- and blue-sideband transitions before the adiabatic transfer are shown in (c) and (d), respectively. Each point obtained is the average of 50 experiments, and the red curves are the results of fitting with multiple Gaussians. The label $-\omega_{\text {c.m. }}$. $\left(+\omega_{\text {c.m. }}\right)$ indicates the red- (blue-) sideband resonance of the center-of-mass (c.m.) mode, and $-\omega_{\text {rock }}\left(+\omega_{\text {rock }}\right)$ the red- (blue-) sideband resonance of the rocking mode. (e),(f) Measurement of the average phonon numbers of collective modes after the adiabatic transfer, in a similar way to (c) and (d). Results of spectroscopy over radial red- and blue-sideband transitions after the adiabatic transfer are shown in (e) and (f), respectively.

adiabatic transfer is realized by shining the excitation laser and sweeping its detuning $\Delta$ over the red-sideband resonance from negative to positive values. The amplitude of the beam is also modulated in a Gaussian shape to ensure 
that $|\Delta| / g$ is large at the beginning and end of the pulse so that the overlap of the initial (final) state and $\left|\psi_{\text {atI }}\right\rangle$ $\left(\left|\psi_{\mathrm{phSF}}\right\rangle\right)$ is optimized. The explicit values of the parameters are as follows. $\Delta / 2 \pi$ is linearly swept from -41 to $59 \mathrm{kHz}$ in $960 \mu \mathrm{s}$, and the JC coupling coefficient $2 g / 2 \pi$ is varied from $0.29 \times 14$ to $14 \mathrm{kHz}$ and back to $0.29 \times$ $14 \mathrm{kHz}$ in a Gaussian shape over the same period [see the inset of Fig. 3(a)]. The red curve in Fig. 3(a) is a numerically simulated result.

The initial population in Fig. 3(a) is $\sim 5 \%$ smaller than what is expected for $\left|\psi_{\text {atI }}\right\rangle$. This is the result of infidelity in the carrier $\pi$ pulse used to prepare $\left|\psi_{\text {att }}\right\rangle$, which is presumably due to jitter in the excitation beam. The final population is floating from zero by $\sim 10 \%$. In addition to the imperfect initialization mentioned above, this is due also to infidelity in the adiabatic transfer process itself, which we speculate is due mainly to the effect of laser frequency fluctuations. We previously analyzed the effects of laser frequency fluctuations and adiabaticity in the rapid adiabatic passage on the sideband transitions (see Fig. 4 of Ref. [25]; although this analysis was done for a single ion, the overall qualitative and quantitative behavior should be similar). We have confirmed in a numerical simulation that the population goes to near zero with less than $1 \%$ error under the assumption of no laser frequency fluctuation. Hence we speculate that the effect of diabatic transitions is limited to below $1 \%$.

We also analyzed the adiabaticity during the transfer process using the theory of adiabatic variations of Hamiltonians [26]. Figure 3(b) shows the time-dependent eigenenergies obtained by diagonalizing the instantaneous Hamiltonians based on the pulse parameters used in the experiment. From these eigenenergies and eigenvectors, the probability of diabatic transitions is estimated in a similar way as in Ref. [25]. The largest leakage from the ground state is the one towards the third lowest level, and its probability is at most $2 \%$. This is consistent with the numerical result given above.

The effect of the adiabatic transfer process on the phonon states is also examined. Figures 3(c)-3(f) show the result of phonon-number measurements. Figures 3(c) and 3(d) show the results of spectroscopy over the radial red- and bluesideband transitions, respectively, at the beginning of the adiabatic transfer process, and Figs. 3(e) and 3(f) show the corresponding results at the end of the process. From these results, the average phonon numbers for the c.m. and rocking modes at the beginning and end are estimated to be $\left(\bar{n}_{\text {c.m. }}, \bar{n}_{\text {rock }}\right)=(0.09 \pm 0.04,0.04 \pm 0.05)$ and $\left(\bar{n}_{\text {c.m. }}, \bar{n}_{\text {rock }}\right)=$ $(0.15 \pm 0.11,1.58 \pm 0.60)$, respectively. At the beginning, both of the phonon modes are almost in the ground states, while at the end, a number of rocking-mode phonon quanta close to 2 is realized and the c.m. mode is almost intact. The above results support the occurrence of a quantum phase transition from the atomic insulator ground state $\left|\psi_{\text {atI }}\right\rangle=\left|e_{1}\right\rangle\left|e_{2}\right\rangle|0\rangle_{1}|0\rangle_{2}$ to the phonon SF ground state $\left|\psi_{\mathrm{phSF}}\right\rangle \equiv\left|g_{1}\right\rangle\left|g_{2}\right\rangle \otimes(1 / \sqrt{2}) \hat{a}_{r}^{\dagger 2}|0\rangle_{1}|0\rangle_{2}$.
The transfer process is further analyzed by estimating the excitation number variances (atomic, phonon, and total) introduced above. The red circles in Fig. 4(a) show the atomic excitation number variance $\Delta \hat{N}_{a, 1}^{2}$ estimated from atomic populations measured with the photomultiplier tube. The peak at the center indicates the presence of polaritons. The numerically simulated results are also shown as the red solid curve. The cause for the discrepancy between the experimental and calculated values is expected to be similar to that discussed in relation to Fig. 3(a). The blue triangles in Fig. 4(a) show the values of the phonon-number variance $\Delta \hat{N}_{p, 1}^{2}$ with $\hat{N}_{p, j}=\hat{a}_{j}^{\dagger} \hat{a}_{j}$, which are obtained by measuring the average phonon numbers in the same way as in Figs. 3(c)-3(f), and estimating the variance according to Eqs. (3) and (4) of the Supplemental Material [27]. This supports the argument that the phonon SF ground state is realized at the end of the adiabatic transfer.

Estimation of the total excitation number variance $\Delta \hat{N}_{1}^{2}$ requires simultaneous measurements of internal and phonon states, which are relatively difficult to perform since

(a)

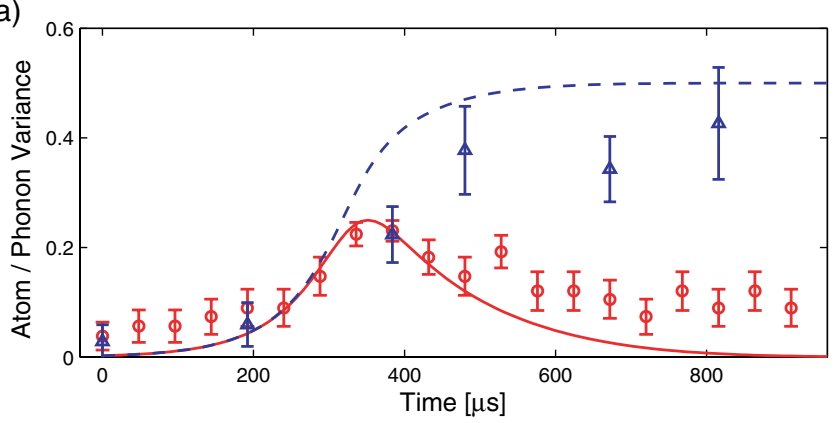

(b)

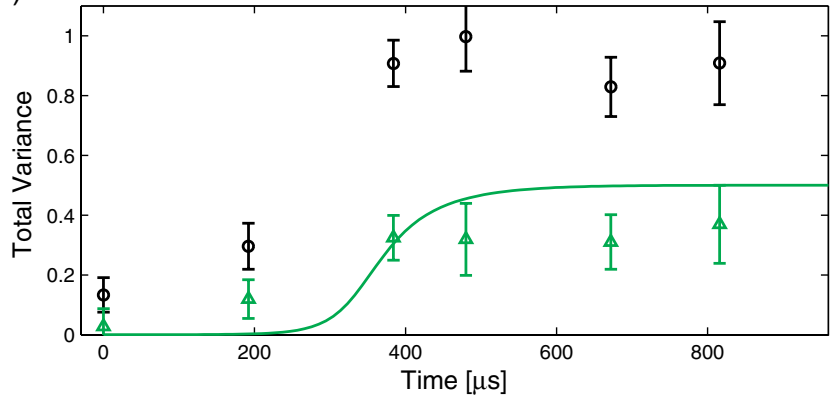

FIG. 4 (color online). Estimated experimental and calculated values for the excitation number variances (atomic, phonon, and total) during the adiabatic transfer. The points are experimental values and the curves are calculated from exact ground states against the actual time dependence of the experimental parameters. (a) Values for the atomic excitation number variance $\Delta \hat{N}_{a, 1}^{2}$ (the circles and the solid curve) and the phonon-number variance $\Delta \hat{N}_{p, 1}^{2}$ (the triangles and the dashed curve). Each point obtained is the average of 50 experiments. (b) Values for the total excitation number variance $\Delta \hat{N}_{1}^{2}$. Instead of directly estimating the experimental values for this quantity, the upper and lower bounds are estimated along with their errors, and shown as the circles and triangles (see text for details). 
phonon states should be once mapped to internal states to be read. We avoid such measurements here and instead estimate $\Delta \hat{N}_{1}^{2}$ from the known quantities $\Delta \hat{N}_{a, 1}^{2}$ and $\Delta \hat{N}_{p, 1}^{2}$. It should be noted that in this case $\Delta \hat{N}_{1}^{2}$ can only be estimated as intervals with upper and lower bounds. The details of the derivation of inequalities for estimating the upper and lower bounds of $\Delta \hat{N}_{1}^{2}$ are given in the Supplemental Material [27].

Figure $4(\mathrm{~b})$ is the total excitation number variance $\Delta \hat{N}_{1}^{2}$. The values (upper and lower bounds) are obtained using Eqs. (5) and (6) of the Supplemental Material[27] along with the results in Fig. 4(a). The expected qualitative behavior, including the onset of a phase transition (near $400 \mu \mathrm{s}$ ), an example of which is seen in Fig. 2 of Ref. [5], is reproduced in these results.

In summary, we have observed dynamics and adiabatic transfer between ground states of a JCH system with two ions. Scaling up the JCH system described in this Letter to include larger numbers of sites necessitates certain points to be overcome. When the number of ions in the linear chain $N_{\text {ions }}$ is increased, the spacing at the center $d_{0}$ decreases in proportion to $\left(N_{\text {ions }}\right)^{-0.559}$ [24] and hence $\kappa$ increases in proportion to $\left(N_{\text {ions }}\right)^{1.677}$. On the other hand, it is desired to keep $\kappa / g$ at moderate values so that the rich phase diagram of the JCH system should be explored as widely as possible. This demand may be fulfilled by tightening the radial confinement (note that $\kappa \propto \omega_{x}^{-1}$ ) or by using an array of independent traps, for which interion distances and magnitudes of confinement can be chosen independently.

This work was supported by the Ministry of Education, Culture, Sports, Science and Technology (MEXT) of Japan, Kakenhi "Quantum Cybernetics" project, and by the Japan Society for the Promotion of Science (JSPS) through its Funding Program for World-Leading Innovative R\&D on Science and Technology (FIRST Program).

*toyoda@ee.es.osaka-u.ac.jp.

†Present address: Institute for Laser Science, University of Electro-Communications, 1-5-1 Chofugaoka, Chofu, Tokyo 182-8585, Japan.

[1] E. T. Jaynes and F. W. Cummings, Proc. IEEE 51, 89 (1963).

[2] D. M. Meekhof, C. Monroe, B. E. King, W. M. Itano, and D. J. Wineland, Phys. Rev. Lett. 76, 1796 (1996).

[3] A. D. Greentree, C. Tahan, J.H. Cole, and L.C. L. Hollenberg, Nat. Phys. 2, 856 (2006).
[4] M. J. Hartmann, F. Brandao, and M. B. Plenio, Nat. Phys. 2, 849 (2006).

[5] D. G. Angelakis, M. F. Santos, and S. Bose, Phys. Rev. A 76, 031805 (2007).

[6] D. Rossini and R. Fazio, Phys. Rev. Lett. 99, 186401 (2007).

[7] E. K. Irish, C. D. Ogden, and M. S. Kim, Phys. Rev. A 77, 033801 (2008).

[8] M. I. Makin, J. H. Cole, C. Tahan, L. C. L. Hollenberg, and A. D. Greentree, Phys. Rev. A 77, 053819 (2008).

[9] P. A. Ivanov, S. S. Ivanov, N. V. Vitanov, A. Mering, M. Fleischhauer, and K. Singer, Phys. Rev. A 80, 060301 (2009).

[10] J. Hubbard, Proc. R. Soc. A 276, 238 (1963).

[11] H. S. J. van der Zant, F. C. Fritschy, W. J. Elion, L. J. Geerligs, and J.E. Mooij, Phys. Rev. Lett. 69, 2971 (1992).

[12] M. Greiner, O. Mandel, T. Esslinger, T. W. Hansch, and I. Bloch, Nature (London) 415, 39 (2002).

[13] S. Rebic, A. S. Parkins, and S. M. Tan, Phys. Rev. A 65, 063804 (2002).

[14] R. P. Feynman, Int. J. Theor. Phys. 21, 467 (1982).

[15] M. Johanning, A. F. Varon, and C. Wunderlich, J. Phys. B 42, 154009 (2009).

[16] R. Blatt and C. F. Roos, Nat. Phys. 8, 277 (2012).

[17] A. Friedenauer, H. Schmitz, J. T. Glueckert, D. Porras, and T. Schaetz, Nat. Phys. 4, 757 (2008).

[18] R. Gerritsma, G. Kirchmair, F. Zahringer, E. Solano, R. Blatt, and C.F. Roos, Nature (London) 463, 68 (2010).

[19] K. Kim, M.S. Chang, S. Korenblit, R. Islam, E. E. Edwards, J. K. Freericks, G. D. Lin, L. M. Duan, and C. Monroe, Nature (London) 465, 590 (2010).

[20] B.P. Lanyon, C. Hempel, D. Nigg, M. Muller, R. Gerritsma, F. Zahringer, P. Schindler, J. T. Barreiro, M. Rambach, G. Kirchmair et al., Science 334, 57 (2011).

[21] R. Islam, E. E. Edwards, K. Kim, S. Korenblit, C. Noh, H. Carmichael, G. D. Lin, L. M. Duan, C. C. J. Wang, J. K. Freericks et al., Nat. Commun. 2, 377 (2011).

[22] D. Porras and J. I. Cirac, Phys. Rev. Lett. 93, 263602 (2004).

[23] S. Haze, Y. Tateishi, A. Noguchi, K. Toyoda, and S. Urabe, Phys. Rev. A 85, 031401 (2012).

[24] D. F. V. James, Appl. Phys. B 66, 181 (1998).

[25] T. Watanabe, S. Nomura, K. Toyoda, and S. Urabe, Phys. Rev. A 84, 033412 (2011).

[26] A. Messiah, Quantum Mechanics (North-Holland, Amsterdam, 1961), Vol. 2.

[27] See Supplemental Material http://link.aps.org/ supplemental/10.1103/PhysRevLett.111.160501 for the details of the estimation of the phonon number and total number variances. 